\title{
Utilización de compost agotado de champiñón como capa de coberturas en nuevos ciclos de producción
}

\author{
Arturo Pardo-Giménez ${ }^{(1)}$, Diego Cunha Zied ${ }^{(2)}$ y José Emilio Pardo-González ${ }^{(3)}$
}

\begin{abstract}
${ }^{(1)}$ Centro de Investigación, Experimentación y Servicios del Champiñón, C/Peñicas, s/no Apartado 63, 16220 Quintanar del Rey (Cuenca), España. E-mail: apardo.cies@dipucuenca.es (2)Universidade Estadual Paulista, Departamento de Produção Vegetal, Faculdade de Ciências Agronômicas, Módulo de Cogumelos, Fazenda Lageado, Rua José Barbosa de Barros, 1.780, Caixa Postal 237, CEP 18603-970 Botucatu, SP, Brasil. E-mail: dczied@fca.unesp.br ${ }^{(3)}$ Universidad de Castilla-La Mancha, Escuela Técnica Superior de Ingenieros Agrónomos, Campus Universitario, s/nº, 02071 Albacete, España. E-mail: jose.pgonzalez@uclm.es
\end{abstract}

Resumen - El objetivo de este trabajo fue evaluar el comportamiento agronómico de seis mezclas de cobertura, elaboradas a partir de sustrato postcultivo del champiñón Agaricus bisporus, en nuevos ciclos de cultivo. Los resultados obtenidos mostraron la viabilidad de la reintroducción del sustrato en nuevos ciclos de cultivo, ya sea como material de base único, si se somete a un proceso de lavado para eliminar sales solubles, o bien mezclado con otros materiales de baja conductividad, como es el caso de la turba rubia o la fibra de coco. Se destacan los altos valores de eficiencia biológica registrados, que llegaron hasta los $100 \mathrm{~kg} \mathrm{~kg}^{-1} \mathrm{de}$ compost, similares a los proporcionados por los testigos, y los altos valores relativos observados (con respecto a los testigos) en el contenido en materia seca de los carpóforos cosechados con algunas de las nuevas coberturas elaboradas. Esta reutilización del compost constituye una alternativa interesante, con vistas a reemplazar a las tierras y a los sustratos orgánicos utilizados habitualmente como cobertura, con la doble ventaja de disminuir los costos de elaboración y el impacto ambiental.

Termos para indexación: Agaricus bisporus, compost, materiales de cobertura alternativos, valorización.

\section{Using spent mushroom substrate as casing layers in new growing cycles}

\begin{abstract}
The objective of this work was to evaluate the agronomic behaviour of six mixtures of casing, prepared from spent mushroom substrate (SMS), in new production cycles of Agaricus bisporus. The results obtained showed the feasibility of reuse of the SMS in new cultivation cycles when used alone by submitting it to a washing process to remove soluble salts, or mixed with other materials of low conductivity, such as Sphagnum peat or coconut fiber pith. The high values of biological efficiency recorded up to $100 \mathrm{~kg} \mathrm{~kg}^{-1}$ compost, similar to that of the controls, and the same high values observed in the dry matter content of the mushrooms harvested with some of the new casing layers prepared must be highlighted. The suggested use for SMS is an important alternative to consider, in order to replace soils and other organic substrates typically used as casing, with the added advantages of lowering the production costs and decreasing the environmental impact.
\end{abstract}

Index terms: Agaricus bisporus, compost, alternative casing layer, valorization.

\section{Introducción}

La cobertura constituye el material empleado como recubrimiento superior de compost, en el que se produce la fructificación del champiñón en cultivo comercial. Muchos son los materiales que, solos o en combinación, han sido empleados como coberturas para cultivo de champiñón, tanto a nivel comercial como a nivel experimental, aunque solo algunos de ellos tengan actualmente aplicación en la práctica. El comportamiento del material en rendimiento y calidad, su disponibilidad y su precio son los factores determinantes en la elección de la cobertura. Junto a distintos tipos de turbas, el empleo de materiales naturales de origen mineral, como diferentes tipos de tierras, gravilla y toba, carbonato cálcico en diferentes formas y sustrato postcultivo es el más extendido en todo el mundo (Pardo et al., 1999).

Actualmente, en España, se necesitan en torno de $1,5 \times 10^{5} \mathrm{~m}^{3}$ anuales de sustratos de cobertura. En la zona productora de champiñón de Castilla-La Mancha, se concentra cerca de $45 \%$ de la producción nacional. Las coberturas empleadas todavía se fundamentan en el uso de suelo mineral de diferentes orígenes (capa arable, subsuelo) como material de base, al que normalmente se adicionan diversos complementos, principalmente 
turbas, como correctores estructurales y de retención de agua (Pardo et al., 1999). La problemática asociada al empleo de suelo mineral (variabilidad de estructura, escasa porosidad y retención de agua, y dificultades para comercializar el compost agotado) y de turbas (principalmente agotamiento de reservas y alteración de ecosistemas) implica la necesidad de buscar alternativas.

Entretanto, las actuales aplicaciones de los sustratos postcultivos de champiñón y setas (Pleurotus spp.), principalmente como componentes de enmiendas y sustratos de cultivo, no parecen suficientes para dar salida al elevado volumen de material generado año tras año, que se acumula en los centros de recogida situados en las zonas productoras y que constituye un contaminante potencial. El sector productor genera, en España, unas $5 \times 10^{5}$ toneladas anuales de residuos de sustratos degradados por los hongos tras el cultivo comercial, mientras que el conjunto de la Unión Europea produce más de 3,5 x $10^{6}$ toneladas anuales. Entre los posibles usos que puede tener el sustrato postcultivo de hongos cultivados encontramos, entre otros, su empleo en biorremediación (purificación de aire, agua, suelos, y de sustratos contaminados con plaguicidas), utilización en otros cultivos (flores y hortalizas en invernadero, frutas y hortalizas en campo, otros cultivos), enmienda general de suelos, semilleros y paisajismo, alimentación animal y acuicultura, control de plagas y enfermedades, y usos diversos (combustible, vermicultura, otros), siendo posible también su reutilización en el cultivo de hongos, como material de cobertura para Agaricus spp. y como sustrato para el cultivo de otras especies (Rinker, 2002).

Con relación a la elaboración de mezclas de cobertura basadas en sustrato postcultivo, revisadas recientemente por Pardo \& Pardo (2008), son numerosas las fórmulas utilizadas en el cultivo comercial y experimental, con diferentes grados de éxito. Entre ellas se destacan algunas con cierto nivel de aplicación en la práctica: una mezcla de tierra, toba volcánica y sustrato postcultivo madurado durante dos años (Sinden, 1971), sustrato postcultivo de un año con arena y cal apagada en proporción 4:1:1 (Mantel, 1973), una mezcla de sustrato postcultivo de más de dos años y piedra caliza molida (Brosius, 1981) y combinaciones de estiércol compostado y sustrato postcultivo de champiñón de dos años en proporción 2:1 (Singh et al., 2000) y 3:1 (Dhar et al., 2003).
Riahi y Arab (2004) evaluaron positivamente en Irán diferentes mezclas de cobertura basadas en sustrato postcultivo de champiñón (SPC) madurado y lavado, con rendimientos entre 206 y $252 \mathrm{~kg}$ de champiñón por tonelada de compost. Resultados similares fueron registrados posteriormente por Riahi y Zamani (2008) utilizando SPC lavado en combinación con turba y azolla compostada, con rendimientos entre $200 \mathrm{y}$ $217 \mathrm{~kg}$ de champiñón por tonelada de compost. En la misma línea de trabajo, Barry et al. (2008) evaluaron en Irlanda coberturas basadas en SPC lavado, mezclado a dosis entre el 10 y el $20 \% \mathrm{v} / \mathrm{v}$ con dos tipos de turba, y encontraron también, para algunas de ellas, resultados comparables a los obtenidos con coberturas comerciales en términos de rendimiento, con promedios entre 250 y $261 \mathrm{~kg}$ de champiñón por tonelada de compost de acuerdo con la proporción de SPC utilizado. En España, Pérez-Clavijo et al. (2009) obtuvieron recientemente valores de eficiencia biológica por encima de $100 \mathrm{~kg} 100 \mathrm{~kg}^{-1}$ compost cuando utilizaron coberturas basadas en mezclas de SPC con turbas.

Si se pretende emplear el material como cobertura, la alta concentración de sales solubles constituye una problemática que implica en la necesidad de realizar un tratamiento de lavado, como paso previo a su utilización, o su mezcla con otros materiales de baja conductividad eléctrica, posibilidades estudiadas previamente en experimentos independientes. Así, Pardo \& Pardo (2008) estudiaron a escala experimental el comportamiento de coberturas basadas en sustrato postcultivo de champiñón (SPC), sometido a diferentes niveles de lavado.

Como continuación del experimento anteriormente citado, y a partir de los resultados en él obtenidos, el objetivo del presente trabajo fue el estudio comparativo de las respuestas agronómicas del champiñón Agaricus bisporus (Lange) Imbach, obtenidas de la evaluación de diferentes sustratos de cobertura, formulados con SPC como material de base, solo (sometido a diferentes niveles de lavado) y en combinación con turba rubia y fibra de coco.

\section{Materiales y Métodos}

Para la caracterización física, química y biológica de los composts y las mezclas de cobertura, se realizaron las siguientes determinaciones: humedad, nitrógeno total, materia orgánica, densidad aparente, carbonatos

Pesq. agropec. bras., Brasília, v.45, n.10, p.1164-1171, out. 2010 
y caliza activa y cenizas (Ministerio de Agricultura, Pesca y Alimentación, 1994), $\mathrm{pH}$, conductividad eléctrica, porosidad total y capacidad de retención de agua (Ansorena, 1994; Asociación Española de Normalización y Certificación, 2001), relación C:N, densidad real (Asociación Española de Normalización y Certificación, 2001), prospección de nematodos (Nombela y Bello, 1983), ácaros (Krantz, 1986) y hongos competidores (Tello et al., 1991).

Como sustrato de base para el cultivo de champiñón, se utilizó un compost comercial basado en paja de trigo y estiércol de pollo. Las características analíticas fueron las siguientes: humedad, $733 \mathrm{~g} \mathrm{~kg}^{-1}$; materia orgánica, $792,1 \mathrm{~g} \mathrm{~kg}^{-1}$; nitrógeno total, 22,6 $\mathrm{g} \mathrm{kg}^{-1}$; relación $\mathrm{C} / \mathrm{N}$, 20,3; pH, 8,09; y contenido en cenizas, $207,9 \mathrm{~g} \mathrm{~kg}^{-1}$. Todos los valores observados se ajustaron al rango considerado como óptimo para el cultivo de champiñón (Hearne, 1994). Fue utilizado, en el presente trabajo, el micelio Fungisem H-25 (híbrido blanco liso), en la razón de $10 \mathrm{~g} \mathrm{~kg}^{-1}$.

En el Cuadro 1, se presenta la composición de las diferentes capas de cobertura evaluadas. Como testigos, se utilizaron cuatro tipos diferentes de cobertura. El primero, del tipo de las mezclas que se utilizan actualmente en el sector productor de Castilla-La Mancha (España), estaba formado por una mezcla de suelo mineral y fibra de coco en proporción 4:1 (v/v) (testigo $\mathrm{SM}+\mathrm{FC}$ ). El segundo es una alternativa sin tierra, más parecida a las que se utilizan habitualmente en países como Holanda, con mayor porosidad y capacidad de retención de agua, formado por una mezcla de turba rubia y carbonato cálcico precipitado de azucarera, en proporción 2:1 (v/v) (testigo TR+CCPA). Se incluyeron también como testigos dos coberturas comerciales basadas en turba, una de origen holandés
(Topterra) y otra de origen español (Torreblanca). Todas estas coberturas presentan un buen comportamiento agronómico previamente demostrado (Pardo et al., 2002).

Para el resto de coberturas, se empleó SPC como material de base. Antes de preparar las mezclas, el SPC utilizado (en el que se incluía la capa de cobertura basada en suelo mineral) fue sometido a un tratamiento térmico $\left(70^{\circ} \mathrm{C}, 12\right.$ horas), previo al vaciado de la sala de cultivo, y a un posterior madurado durante dos meses, de acuerdo con Lohr et al. (1984).

En la preparación de las coberturas 5 y 6 , se utilizó SPC con dos niveles de lavado. Para ello se colocaron aproximadamente $40 \mathrm{~L}$ del SPC en un cajón, con una malla en el fondo a una altura de $40 \mathrm{~cm}$, y se fueron añadiendo, por la parte superior, cantidades de $10 \mathrm{~L}$ de agua desionizada a intervalos de 5-10 min. Así, para la preparación de la cobertura 5 (SPC L1), se lavaron $40 \mathrm{~L}$ de sustrato con $40 \mathrm{~L}$ de agua, mientras para la cobertura 6 (SPC L2) fueron utilizados $80 \mathrm{~L}$ de agua.

Otras dos coberturas ( 7 y 8 , Cuadro 1 ) se prepararon con turba rubia mezclada con SPC en proporciones $4: 1$ y $3: 2(\mathrm{v} / \mathrm{v})$. De la misma manera se prepararon otras dos coberturas, habiéndose mezclado fibra de coco y SPC en proporciones 4:1 y 3:2 (v/v) (coberturas 9 y 10). A todas las coberturas basadas en SPC se les añadió carbonato cálcico a razón de $100 \mathrm{~g} \mathrm{~L}^{-1}$.

Se utilizó un diseño de bloques al azar, con seis repeticiones y 10 tratamientos, lo que resultó en un total de 60 cubetas, cada una con $6 \mathrm{~kg}$ de compost. El espesor de cobertura empleado añadido fue de $3 \mathrm{~cm}$.

La conducción del ciclo de cultivo se llevó a cabo en las condiciones de temperatura, humedad relativa y concentración de dióxido de carbono recomendadas para la variedad comercial de micelio seleccionada.

Cuadro 1. Composición de las mezclas de cobertura.

\begin{tabular}{lll}
\hline Cobertura & \multicolumn{1}{c}{ Identificación } & \multicolumn{1}{c}{ Composición } \\
\hline 1 & Testigo SM+FC & Suelo mineral + fibra de coco 4:1 (v/v) \\
2 & Testigo TR+CCPA & Turba rubia + carbonato cálcico precipitado de azucarera 2:1 (v/v) \\
3 & Testigo Topterra & Cobertura comercial \\
4 & Testigo Torreblanca & Cobertura comercial \\
5 & SPC L1 & Sustrato postcultivo de champiñón lavado con 1 volumen de agua $\left(+100 \mathrm{gL}^{-1} \mathrm{CaCO}_{3}\right)$ \\
6 & SPC L2 & Sustrato postcultivo de champiñón lavado con 2 volúmenes de agua $\left(+100 \mathrm{gL}^{-1} \mathrm{CaCO}_{3}\right)$ \\
7 & TR-SPC $4: 1$ & Turba rubia + sustrato postcultivo de champiñón $4: 1(\mathrm{v} / \mathrm{v})\left(+100 \mathrm{gL}^{-1} \mathrm{CaCO}_{3}\right)$ \\
8 & TR-SPC $3: 2$ & Turba rubia + sustrato postcultivo de champiñón $3: 2(\mathrm{v} / \mathrm{v})\left(+100 \mathrm{gL}^{-1} \mathrm{CaCO}_{3}\right)$ \\
9 & FC-SPC $4: 1$ & Fibra de coco + sustrato postcultivo de champiñón $4: 1(\mathrm{v} / \mathrm{v})\left(+100 \mathrm{gL}^{-1} \mathrm{CaCO}_{3}\right)$ \\
10 & FC-SPC $3: 2$ & Fibra de coco + sustrato postcultivo de champiñón $3: 2(\mathrm{v} / \mathrm{v})\left(+100 \mathrm{gL}^{-1} \mathrm{CaCO}_{3}\right)$
\end{tabular}


Durantela fase de crecimiento vegetativo, la temperatura fue de $24^{\circ} \mathrm{C}$, la concentración de $\mathrm{CO}_{2}>2.000 \mathrm{ppm}$ y la humedad relativa del 95\%, sin ventilación. Para la inducción de la fructificación, se bajaron la temperatura, el contenido en $\mathrm{CO}_{2}$ y la humedad relativa a $18^{\circ} \mathrm{C}$, $1.000 \mathrm{ppm}$ y $87 \%$, respectivamente, condiciones que se mantuvieron durante la fase de producción. La capa de cobertura se aplicó tras un periodo de germinación de 14 días, tras lo cual se aplicaron los tratamientos fitosanitarios habituales, como: formalina, diflubenzuron y prochloraz. Las coberturas se rastrillaron 7 días después de su aplicación, y dos días después se procedió a inducir la fructificación. La duración total de ciclo de cultivo fue de 63 días, y se cosecharon cuatro floradas.

La recolección de los champiñones se realizó diariamente, en el estadoóptimo comercial de desarrollo, correspondiente a los estados morfogenéticos 2, 3 y 4 de los definidos por Hammond \& Nichols (1976). Para el establecimiento del rendimiento por unidad de superficie, se pesó la cantidad de champiñones producidos diariamente y se contó el número de champiñones obtenidos. Además, se separó la producción en dos grupos según su tamaño: gruesos ( $\geq 40 \mathrm{~mm}$ ) y medianos $(15-40 \mathrm{~mm})$. La eficiencia biológica, expresada en kg hongos cosechados $100 \mathrm{~kg}^{-1}$ compost (materia seca), se estableció a partir del rendimiento por unidad de superficie.

El peso unitario de los champiñones se determinó a partir del rendimiento obtenido y del número de champiñones cosechados. Una segunda estimación del tamaño, expresado como diámetro del sombrero en milímetros, se determinó con las curvas de regresión no lineal previamente establecidas, a partir de la determinación del diámetro y del peso de champiñones correspondientes a las tres primeras floradas, de la variedad comercial de micelio seleccionada.

En el día de la máxima cosecha de cada una de las tres primeras floradas, se eligieron champiñones de tamaño y madurez uniformes para la evaluación de su calidad. Sobre ellos se determinó color, contenido en materia seca y textura. La precocidad se expresó como el tiempo transcurrido entre la aplicación de la cobertura y la cosecha de la primera florada.

El color de la superficie de los champiñones se midió por reflexión, con un colorímetro marca Minolta, en un total de veinte medidas en cada una de las tres primeras floradas para cada cubeta, habiéndo-se realizádo cuatro medidas sobre los sombreros de cinco champiñones, de tamaño uniforme y libres de enfermedad. Para la descripción del color, se utilizaron los valores de $L^{*}$ (luminosidad), $b^{*}$ (componente amarillo-azul) e $\Delta \mathrm{E}^{*}$, parámetro que mide el grado de desviación, en comparación con los valores de un esporóforo de color ideal (Pardo et al., 1999).

El método utilizado para determinar el contenido de materia seca de los carpóforos consistió en la medida de la pérdida de peso, tras desecación a $105^{\circ} \mathrm{C}$ durante 72 horas (Lau, 1982). Para evaluar la textura, se utilizó un analizador TA-XT Plus (Stable Micro Systems Godalming, UK), y se cortó el pie del champiñon perpendicularmente a nivel del velo, para medir la textura sobre el centro del sombrero, con una punta de prueba cilíndrica de acero inoxidable, en una velocidad constante de ensayo de $2 \mathrm{~mm} \mathrm{~s}^{-1}$.

Para la realización del análisis estadístico, se utilizó el paquete informático Statgraphics Plus v. 4.1 (Statistical Graphics Corp., Princeton, USA). Se empleó la técnica de análisis de varianza para evaluar los datos. Para el establecimiento de diferencias significativas entre medias, se utilizó el test de Tukey-HSD al 5\% de probabilidad.

\section{Resultados y Discusión}

En el Cuadro 2, se presentan las características físicas, químicas y biológicas de los diferentes materiales de cobertura ensayados.

En el momento de aplicación de las coberturas, el contenido en humedad de las mismas alcanzó una media de $538 \mathrm{~g} \mathrm{~kg}^{-1}$, siendo el menor el del testigo SM+FC (213 $\left.\mathrm{g} \mathrm{kg}^{-1}\right)$ y el mayor del testigo Topterra $\left(749 \mathrm{~g} \mathrm{~kg}^{-1}\right)$.

Con respecto al $\mathrm{pH}$, los resultados analíticos muestraron ligeras diferencias, que oscilaron entre el 7,64 del testigo Torreblanca y 8,39 del testigo SM+FC, con un promedio de 7,98.

En cuanto a la conductividad eléctrica de las coberturas estudiadas, se observaron, en todos los casos, valores superiores a los testigos, con un valor máximo de $1.616 \mu \mathrm{S} \mathrm{cm}^{-1}$ para la cobertura en la que se utilizaba sustrato postcultivo lavado con un volumen de agua.

Los valores registrados para la densidad aparente y la densidad real de las coberturas utilizadas se encuentran entre los observados para los testigos, y alcanzaron - en las basadas en sustrato postcultivo - valores 
de porosidad entre 819 y $912 \mathrm{~mL} \mathrm{~L}^{-1}$, sensiblemente superiores al testigo basado en suelo mineral (719 $\left.\mathrm{mL} \mathrm{L}^{-1}\right)$ e inferiores, aunque más próximos, al testigo Topterra $\left(924 \mathrm{~mL} \mathrm{~L}^{-1}\right)$.

En cuanto a la capacidad de retención de agua de las mezclas analizadas, se observó una situación similar a la observada con la porosidad, aunque con marcadas diferencias, con valores entre 1,01 y $3,47 \mathrm{~kg} \mathrm{~kg}^{-1}$ (SPC L1 y FC-SPC 4:1, respectivamente); todas las mezclas analizadas fueron superiores al testigo basado en suelo mineral $\left(0,63 \mathrm{~kg} \mathrm{~kg}^{-1}\right)$ e inferiores al testigo Topterra $\left(5,07 \mathrm{~kg} \mathrm{~kg}^{-1}\right)$.

Los parámetros porosidad y capacidad de retención de agua son de especial interés, si se tienen en cuenta las funciones que debe cumplir la capa de cobertura. Por un lado, una alta porosidad facilita la fructificación al favorecer el intercambio gaseoso, mientras que interesan altos valores de la capacidad de retención de agua, ya que ésta contribuye a la humedad del microclima donde se produce la fructificación y, por otro lado, junto al compost, aporta agua para el desarrollo de los carpóforos (Pardo et al., 1999). En cuanto al resto de parámetros, los resultados observados para las nuevas coberturas corresponden a las características de los materiales de origen del sustrato postcultivo (compost de base y cobertura mineral) y los otros sustratos empleados para las mezclas, destacándose los valores relativamente altos del contenido en nitrógeno y cenizas de las coberturas con SPC. En estas coberturas, la adición de $\mathrm{CaCO}_{3}$ proporciona altos contenidos en caliza activa, entre 440 y $502 \mathrm{~g} \mathrm{~kg}^{-1}$, lo que, además del aporte de calcio, asegura el efecto tamponador frente a cambios de $\mathrm{pH}$. La prospección de ácaros, nematodos y hongos competidores mostró resultados negativos para todas las coberturas ensayadas.

En el Cuadro 3 se presentan los resultados obtenidos para los principales parámetros de producción cuantitativos evaluados en los champiñones procedentes de las distintas capas de cobertura ensayadas.

Para el número de champiñones cosechados por unidad de superficie, los resultados más bajos fueron proporcionados por las coberturas 8 (TR-SPC 3:2) y 6 (SPC L2), en las que se cosecharon, respectivamente, 1.602 y 1.617 champiñones $\mathrm{m}^{-2}$. No obstante, difirieron de manera significativa únicamente del testigo Torreblanca. Con relación al peso unitario, con una media de $10,7 \mathrm{~g}$, únicamente se observaron diferencias significativas entre dos de los testigos, $\mathrm{SM}+\mathrm{FC}$ y Torreblanca, con 11,9 g y 9,5 g, respectivamente.

Con respecto a la distribución de la producción por floradas, la primera fue en general, la más productiva, seguida de la segunda; en las últimas floradas se observó una notable disminución.

El rendimiento total medio observado en el experimento fue de $18,05 \mathrm{~kg} \mathrm{~m}^{-2}$. El registro más bajo fue obtenido, de manera significativa, con la cobertura 6 , SPC L2 (16,78 $\left.\mathrm{kg} \mathrm{m}^{-2}\right)$, aunque resultó inferior de manera significativa únicamente con respecto al testigo basado en suelo mineral (SM+FC), que proporcionó el mayor rendimiento $\left(19,93 \mathrm{~kg} \mathrm{~m}^{-2}\right)$. Aunque los rendimientos por unidad de superficie no resultaron especialmente altos en el ensayo, los valores de la eficiencia biológica lo fueron, debido a que el compost utilizado presentaba un alto contenido en humedad $\left(733 \mathrm{~g} \mathrm{~kg}^{-1}\right)$. Así, todas

Cuadro 2. Características físicas, químicas y biológicas de los materiales de cobertura ${ }^{(1)}$.

\begin{tabular}{|c|c|c|c|c|c|c|c|c|c|c|c|c|c|c|}
\hline \multirow[t]{2}{*}{ Cobertura $^{(2)}$} & \multirow{2}{*}{$\begin{array}{c}\text { Humedad } \\
\left(\mathrm{g} \mathrm{kg}^{-1}\right)\end{array}$} & \multirow{2}{*}{$\begin{array}{c}\mathrm{pH} \\
(1: 5, \mathrm{v} / \mathrm{v})\end{array}$} & \multirow{2}{*}{$\begin{array}{c}\mathrm{CE} \\
\left(\mu \mathrm{S} \mathrm{cm}^{-1}\right)\end{array}$} & \multicolumn{2}{|c|}{ Densidad aparente } & \multirow{2}{*}{$\begin{array}{c}\text { Densidad } \\
\text { real } \\
\left(\mathrm{g} \mathrm{cm}^{-3}\right) \\
\end{array}$} & \multirow{2}{*}{$\begin{array}{c}\text { Porosidad } \\
\text { total } \\
\left(\mathrm{ml} \mathrm{L}^{-1}\right)\end{array}$} & \multirow{2}{*}{$\begin{array}{c}\text { CRA } \\
\left(\mathrm{kg} \mathrm{kg}^{-1}\right)\end{array}$} & \multirow{2}{*}{$\begin{array}{c}\text { Nitrógeno } \\
\text { total } \\
\left(\mathrm{g} \mathrm{kg}^{-1}\right)\end{array}$} & \multirow{2}{*}{$\begin{array}{l}\text { Cenizas } \\
\left(\mathrm{g} \mathrm{kg}^{-1}\right)\end{array}$} & \multirow{2}{*}{$\begin{array}{c}\text { Materia } \\
\text { orgánica } \\
\left(\mathrm{g} \mathrm{kg}^{-1}\right)\end{array}$} & \multirow{2}{*}{$\begin{array}{c}\text { Carbonatos } \\
\text { totales } \\
\left(\mathrm{g} \mathrm{kg}^{-1}\right)\end{array}$} & \multirow[t]{2}{*}{$\mathrm{C} / \mathrm{N}$} & \multirow{2}{*}{$\begin{array}{l}\text { Caliza } \\
\text { activa }\end{array}$} \\
\hline & & & & $\begin{array}{c}\text { fresco } \\
\left(\mathrm{g} \mathrm{cm}^{-3}\right) \\
\end{array}$ & $\begin{array}{c}\text { seco } \\
\left(\mathrm{g} \mathrm{cm}^{-3}\right)\end{array}$ & & & & & & & & & \\
\hline Testigo SM+FC & 213 & 8,39 & 205 & 0,911 & 0,717 & 2,547 & 719 & 0,63 & 0,3 & 943,3 & 56,7 & 109,6 & 644 & 240 \\
\hline Testigo TR+CCPA & 583 & 8,08 & 831 & 0,644 & 0,269 & 2,258 & 881 & 2,24 & 4,0 & 755,6 & 244,4 & 35,4 & 610 & 238 \\
\hline Testigo Topterra & 749 & 8,17 & 221 & 0,550 & 0,138 & 1,826 & 924 & 5,07 & 7,3 & 364,3 & 635,7 & 50,5 & 191 & 64 \\
\hline Testigo Torreblanca & 596 & 7,64 & 900 & 0,599 & 0,242 & 2,028 & 881 & 2,34 & 1,1 & 567,6 & 432,4 & 22,8 & 338 & 171 \\
\hline SPC L1 & 412 & 7,87 & 1.616 & 0,713 & 0,419 & 2,311 & 819 & 1,01 & 7,2 & 793,6 & 206,4 & 16,6 & 489 & 179 \\
\hline SPC L2 & 417 & 7,78 & 1.306 & 0,703 & 0,410 & 2,310 & 823 & 1,07 & 7,2 & 792,9 & 207,1 & 16,7 & 440 & 154 \\
\hline TR-SPC 4:1 & 601 & 7,89 & 942 & 0,507 & 0,202 & 2,151 & 906 & 2,85 & 5,0 & 673,2 & 326,8 & 37,9 & 502 & 260 \\
\hline TR:SPC 3:2 & 567 & 7,75 & 1.479 & 0,608 & 0,263 & 2,195 & 880 & 2,42 & 5,8 & 708,1 & 291,9 & 29,2 & 492 & 238 \\
\hline FC-SPC 4:1 & 650 & 8,17 & 969 & 0,538 & 0,188 & 2,141 & 912 & 3,47 & 4,4 & 665,2 & 334,8 & 44,1 & 468 & 250 \\
\hline FC-SPC $3: 2$ & 596 & 8,05 & 1.611 & 0,621 & 0,251 & 2,211 & 887 & 2,47 & 5,5 & 719,9 & 280,1 & 29,5 & 468 & 230 \\
\hline Media & 538 & 7,98 & 1.008 & 0,639 & 0,310 & 2,198 & 863 & 2,36 & 4,8 & 698,4 & 301,6 & 39,2 & 464 & 202 \\
\hline
\end{tabular}

${ }^{(1)} \mathrm{CE}$, conductividade eléctrica; $\mathrm{CRA}$, capacidad de retención de agua; $\mathrm{C} / \mathrm{N}$, relación carbono/nitrógeno. ${ }^{(2)} \mathrm{SM}$, suelo mineral; FC, fibra de coco; TR, turba rubia; CCPA, carbonato cálcico precipitado de azucarera; SPC, sustrato postcultivo de champiñón; L, nivel de lavado. 
las coberturas ensayadas, a excepción de la número 6

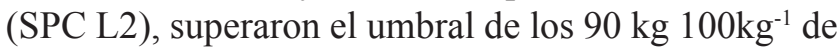
compost.

Para la precocidad, las coberturas basadas en SPC presentaron valores intermedios entre la más precoz (testigo Topterra, 20,7 días) y la que presentó el mayor retraso (testigo $\mathrm{SM}+\mathrm{FC}, 22,1$ días).

En el Cuadro 4, se presentan los resultados obtenidos para los principales parámetros de producción cualitativos, evaluados en los champiñones procedentes de las distintas capas de cobertura ensayadas.

La separación de la producción, según categoría comercial por tamaño, no mostró diferencias significativas entre las coberturas ensayadas, y presentó una mayor proporción de champiñón mediano, con una media de $14,73 \mathrm{~kg} \mathrm{~m}^{-2}$, de que de champiñón grueso $10,28 \mathrm{~kg} \mathrm{~m}^{-2}$.

Al igual del peso unitario, para el diámetro de los carpóforos, con una media de $30,3 \mathrm{~mm}$, únicamente se observaron diferencias significativas entre dos de los testigos ( $\mathrm{SM}+\mathrm{FC}$ con $31,6 \mathrm{~mm}$ y Torreblanca con 29,0 mm), situándose el resto de coberturas entre esos valores.

Para los parámetros que definen el color de los carpóforos, no se observaron diferencias significativas para la luminosidad $\left(\mathrm{L}^{*}\right)$, con una media de 94,12, mientras que para los otros parámetros considerados $\left(\mathrm{b}^{*} \mathrm{e}\right.$ $\left.\Delta \mathrm{E}^{*}\right)$ únicamente resultan destacables los buenos registros proporcionados por los champiñones cosechados que utilizaron la cobertura comercial Topterra.

Cuadro 3. Resultados obtenidos para los principales parámetros de producción cuantitativos, evaluados en los champiñones procedentes de las distintas capas de cobertura ensayadas ${ }^{(1)}$.

\begin{tabular}{|c|c|c|c|c|c|c|c|c|c|}
\hline \multirow[t]{2}{*}{ Cobertura } & \multirow{2}{*}{$\begin{array}{l}\text { Número champiñones } \\
\mathrm{m}^{-2}\end{array}$} & \multirow{2}{*}{$\begin{array}{c}\text { Peso unitario } \\
\text { g por champiñón }\end{array}$} & \multicolumn{5}{|c|}{ Rendimiento $\left(\mathrm{kg} \mathrm{m}^{-2}\right)$} & \multirow{2}{*}{$\begin{array}{l}\text { Eficiencia biológica } \\
\mathrm{kg} 100 \mathrm{~kg}^{-1} \text { compost }\end{array}$} & \multirow{2}{*}{$\begin{array}{l}\text { Precocidad (días } \\
\text { desde la cobertura) }\end{array}$} \\
\hline & & & 1a florada & $2^{\mathrm{a}}$ florada & 3a florada & 4a florada & Total & & \\
\hline Testigo SM+FC & $1.688 \mathrm{ab}$ & $11,9 \mathrm{a}$ & $7,95 \mathrm{a}$ & $5,99 \mathrm{abc}$ & 3,33 & 2,61 & $19,93 \mathrm{a}$ & & \\
\hline Testigo TR+CCPA & $1.724 \mathrm{ab}$ & $10,9 \mathrm{ab}$ & $6,47 b$ & $5,95 \mathrm{abc}$ & 3,20 & 3,21 & $18,82 \mathrm{ab}$ & $104,7 \mathrm{a}$ & $22,1 \mathrm{a}$ \\
\hline Testigo Topterra & $1.630 \mathrm{~b}$ & $10,8 \mathrm{ab}$ & $6,11 b$ & $5,86 \mathrm{abc}$ & 3,25 & 2,39 & $17,61 \mathrm{ab}$ & $98,9 \mathrm{ab}$ & $21,3 \mathrm{abc}$ \\
\hline Testigo Torreblanca & $1.931 \mathrm{a}$ & $9,5 \mathrm{~b}$ & $6,34 b$ & $6,76 \mathrm{a}$ & 2,83 & 2,30 & $18,23 \mathrm{ab}$ & $92,5 \mathrm{ab}$ & $20,7 \mathrm{c}$ \\
\hline SPC L1 & $1.696 \mathrm{ab}$ & $10,1 \mathrm{ab}$ & $6,68 \mathrm{ab}$ & $5,38 \mathrm{abc}$ & 2,84 & 2,23 & $17,13 \mathrm{ab}$ & $95,8 \mathrm{ab}$ & $20,9 b c$ \\
\hline SPC L2 & $1.617 \mathrm{~b}$ & $10,4 \mathrm{ab}$ & $6,70 \mathrm{ab}$ & $5,21 b c$ & 2,61 & 2,27 & $16,78 b$ & $90,0 \mathrm{ab}$ & $21,0 \mathrm{abc}$ \\
\hline TR-SPC 4:1 & $1.724 \mathrm{ab}$ & $11,1 \mathrm{ab}$ & $6,89 \mathrm{ab}$ & $5,78 \mathrm{abc}$ & 3,12 & 3,22 & $19,02 \mathrm{ab}$ & $88,2 b$ & $21,2 \mathrm{abc}$ \\
\hline TR-SPC 3:2 & $1.602 b$ & $10,9 \mathrm{ab}$ & $6,27 b$ & $4,84 \mathrm{c}$ & 3,24 & 2,81 & $17,15 \mathrm{ab}$ & $100,0 \mathrm{ab}$ & $21,1 \mathrm{abc}$ \\
\hline FC-SPC 4:1 & $1.778 \mathrm{ab}$ & $10,5 \mathrm{ab}$ & $6,36 \mathrm{~b}$ & $6,38 \mathrm{ab}$ & 3,41 & 2,53 & $18,68 \mathrm{ab}$ & $90,1 \mathrm{ab}$ & $21,6 \mathrm{abc}$ \\
\hline FC-SPC $3: 2$ & $1.642 \mathrm{ab}$ & $10,5 \mathrm{ab}$ & $6,60 \mathrm{~b}$ & $5,36 \mathrm{abc}$ & 2,76 & 2,45 & $17,16 \mathrm{ab}$ & $98,1 \mathrm{ab}$ & $21,6 \mathrm{abc}$ \\
\hline Media & 1.703 & 10,7 & 6,64 & 5,75 & 3,06 & 2,60 & 18,05 & $90,2 \mathrm{ab}$ & $22,0 \mathrm{ab}$ \\
\hline
\end{tabular}

(1) Para cada columna, valores seguidos de distintas letras son significativamente diferentes entre sí por el test de Tukey al 5\% de probabilidad. SM, suelo mineral; FC, fibra de coco; TR, turba rubia; CCPA, carbonato cálcico precipitado de azucarera; SPC, sustrato postcultivo de champiñón; L, nivel de lavado.

Cuadro 4. Resultados obtenidos en cuanto a los principales parámetros de cualitativos, evaluados en los champiñones procedentes de las distintas capas de cobertura ensayadas ${ }^{(1)}$.

\begin{tabular}{|c|c|c|c|c|c|c|c|c|c|}
\hline \multirow[t]{2}{*}{ Cobertura } & \multicolumn{2}{|c|}{ Categoría comercial $\left(\mathrm{kg} / \mathrm{m}^{2}\right)$} & \multirow{2}{*}{$\begin{array}{c}\text { Diámetro del } \\
\text { carpóforo (mm) }\end{array}$} & \multicolumn{3}{|c|}{ Color } & \multirow{2}{*}{$\begin{array}{c}\text { Materia seca } \\
\left(\mathrm{g} \mathrm{kg}^{-1}\right)\end{array}$} & \multicolumn{2}{|c|}{ Textura } \\
\hline & Grueso $(\geq 40 \mathrm{~mm})$ & Mediano $(<40 \mathrm{~mm})$ & & $\mathrm{L}^{*}$ & $\mathrm{~B}^{*}$ & $\Delta \mathrm{E}^{*}$ & & $\mathrm{~F}_{\text {ruptura }}(\mathrm{N})$ & $\mathrm{E}_{\text {compresión }}(\mathrm{MJ})$ \\
\hline Testigo SM+FC & 3,88 & 16,05 & $31,6 a$ & 93,88 & $9,255 \mathrm{bc}$ & $9,98 \mathrm{ab}$ & $68,1 \mathrm{c}$ & $18,1 \mathrm{~cd}$ & $258,4 \mathrm{ab}$ \\
\hline Testigo TR+CCPA & 4,23 & 14,59 & $30,6 a b$ & 94,25 & $9,299 \mathrm{abc}$ & $9,87 \mathrm{ab}$ & $74,9 \mathrm{ab}$ & $18,9 \mathrm{bcd}$ & $261,0 \mathrm{ab}$ \\
\hline Testigo Topterra & 3,01 & 14,60 & $30,5 \mathrm{ab}$ & 93,81 & $8,410 \mathrm{c}$ & $9,23 \mathrm{~b}$ & $67,0 \mathrm{c}$ & $17,3 \mathrm{~d}$ & $254,1 \mathrm{ab}$ \\
\hline Testigo Torreblanca & 2,74 & 15,50 & $29,0 b$ & 93,74 & $9,599 \mathrm{ab}$ & $10,37 \mathrm{a}$ & $69,8 \mathrm{bc}$ & $19,0 \mathrm{bcd}$ & $243,7 \mathrm{ab}$ \\
\hline SPC L1 & 2,61 & 14,51 & $29,7 \mathrm{ab}$ & 94,27 & $10,199 \mathrm{ab}$ & $10,70 \mathrm{a}$ & $79,2 \mathrm{a}$ & $21,5 \mathrm{a}$ & $269,3 \mathrm{a}$ \\
\hline SPC L2 & 2,89 & 13,89 & $30,0 \mathrm{ab}$ & 94,36 & $10,369 \mathrm{a}$ & $10,82 \mathrm{a}$ & $78,3 \mathrm{a}$ & $21,0 \mathrm{ab}$ & $266,8 \mathrm{ab}$ \\
\hline TR-SPC 4:1 & 4,08 & 14,94 & $30,7 \mathrm{ab}$ & 94,18 & $9,449 \mathrm{abc}$ & $10,04 \mathrm{ab}$ & $71,1 \mathrm{bc}$ & $18,0 \mathrm{~cd}$ & $257,4 \mathrm{ab}$ \\
\hline TR-SPC $3: 2$ & 3,28 & 13,88 & $30,5 \mathrm{ab}$ & 94,21 & $9,837 \mathrm{ab}$ & $10,39 a$ & $78,1 \mathrm{a}$ & $20,4 a b c$ & $262,2 \mathrm{ab}$ \\
\hline FC-SPC 4:1 & 3,79 & 14,89 & $30,1 \mathrm{ab}$ & 94,04 & $9,990 \mathrm{ab}$ & $10,60 \mathrm{a}$ & $70,8 \mathrm{bc}$ & $18,5 \mathrm{bcd}$ & $232,0 \mathrm{~b}$ \\
\hline FC-SPC $3: 2$ & 2,76 & 14,41 & $30,2 \mathrm{ab}$ & 94,46 & $10,135 \mathrm{ab}$ & $10,57 \mathrm{a}$ & $79,7 \mathrm{a}$ & $20,0 \mathrm{abc}$ & $256,0 \mathrm{ab}$ \\
\hline Media & 3,33 & 14,73 & 30,3 & 94,12 & 9,654 & 10,26 & 73,7 & 19,3 & 256,1 \\
\hline
\end{tabular}

(1) Para cada columna, valores seguidos de distintas letras son significativamente diferentes entre sí por el test de Tukey al 5\% de probabilidad. SM, suelo mineral; FC, fibra de coco; TR, turba rubia; CCPA, carbonato cálcico precipitado de azucarera; SPC, sustrato postcultivo de champiñón; L, nivel de lavado. 
El contenido en materia seca de los champiñones es de especial interés, ya que aquellos con alto contenido en materia seca son de textura más firme, de mayor valor nutritivo y tienen menos predisposición al deterioro microbiano (Schisler, 1983; Beelman, 1988). En este estudio, se obtuvieron valores relativamente bajos, con una media de $73,7 \mathrm{~g} \mathrm{~kg}^{-1}$. Los valores más altos, por encima de los cuatro testigos, correspondieron a las coberturas 10 y 8 , basadas en mezclas de fibra de coco y turba rubia con SPC, cuando combinadas en proporción $3: 2(\mathrm{v} / \mathrm{v})\left(79,7\right.$ y $78,1 \mathrm{~g} \mathrm{~kg}^{-1}$, respectivamente) y a las coberturas 5 y 6 , basadas en SPC lavado (79,2 y $78,3 \mathrm{~g} \mathrm{~kg}^{-1}$, respectivamente), aunque no mostraron diferencias significativas con respecto al testigo TR+CCPA $\left(74,9 \mathrm{~g} \mathrm{~kg}^{-1}\right)$. Se observa que altos valores del contenido en materia seca corresponden a altos valores de los parámetros que definen la textura, en particular de la fuerza de ruptura, para la cual los mayores registros se obtuvieron con las coberturas de mayor contenido en materia seca anteriormente citadas.

La conductividad eléctrica parece ser el factor determinante en el comportamiento agronómico de la capa de cobertura, cuando se utiliza SPC como ingrediente. En un trabajo previo con este tipo de materiales (Pardo et al., 2004), se fijó en torno a 1.600 $\mu \mathrm{S} \mathrm{cm}^{-1}$ el umbral a partir del cual cabría esperar disminuciones notables de rendimiento. En el presente trabajo, se han formulado coberturas que ya habían mostrado su potencial en experimentos independientes anteriores y cuya conductividad se situaba en torno a ese valor como límite superior.

\section{Conclusiones}

1. Todas las coberturas utilizadas muestran la viabilidad de la reintroducción del SPC en nuevos ciclos de cultivo, ya sea como material de base único, o bien mezclado con otros materiales.

2. Los sustratos orgánicos utilizados actualmente en la producción comercial pueden ser substituidos parcialmente por reintroducción de SPC.

\section{Agradecimientos}

Al Instituto Nacional de Investigación y Tecnología Agraria y Alimentaria y el Fondo Europeo de Desarrollo Regional, por el apoyo.

\section{Referencias}

ANSORENA, J. Sustratos: propiedades y caracterización. Madrid: Mundi-Prensa, 1994. 172p.

ASOCIACIÓN ESPAÑOLA DE NORMALIZACIÓN Y CERTIFICACIÓN. UNE-EN 13037: mejoradores de suelos y sustratos de cultivo. Madrid: AENOR, 2001. 132p.

BARRY, J.; GRANT, J.; DOYLE, O.; GROGAN, H. Partial substitution of peat with spent mushroom substrate in peat-based casing blends. Mushroom Science, v.17, p.288-309, 2008.

BEELMAN, R.B. Factors influencing post harvest quality and shelf life of fresh mushrooms. Mushroom Journal, v.182, p.455-463, 1988.

BROSIUS, C.C. Spent compost as an alternate casing material. Mushroom Science, v.11, p.397-403, 1981.

DHAR, B.L.; AHLAWAT, O.P.; GUPTA, Y. Evaluation of agro-industrial wastes as casing materials in Agaricus bisporus cultivation in India. Mushrooms International, v.92, p.5-9, 2003.

HAMMOND, J.B.W.; NICHOLS, R. Carbohydrate metabolism in Agaricus bisporus (Lange) Sing: changes in soluble carbohydrates during growth of mycelium and sporophore. Journal of General Microbiology, v.93, p.309-320, 1976.

HEARNE, A.J. Mushroom compost analysis. A few practical suggestions. Mushroom Journal, v.539, p.18-19, 1994.

KRANTZ, G.W. A manual of acarology. $2^{\text {nd }}$ ed. Corvallis: Oregon State University Book Stores, 1986. 509p.

LAU, O. Methods of chemical analysis of mushrooms. In: CHANG, S.T.; QUIMIO, T.H. (Ed.). Tropical mushrooms: biological nature and cultivation methods. Hong Kong: The Chinese University, 1982. p.87-116.

LOHR, V.I.; WANG, S.H.L.; WOLT, J.D. Physical and chemical characteristics of fresh and aged spent mushroom compost. HortScience, v.19, p.681-683, 1984.

MANTEL, E.F.K. Casing soil made from spent compost. Indian Journal of Mushrooms, v.1, p.15-16, 1973.

MINISTERIO DE AGRICULTURA, PESCAY ALIMENTACIÓN. Métodos Oficiales de Análisis. Madrid: MAPA, 1994. 532p.

NOMBELA, G.; BELLO, A. Modificaciones al método de extracción de nematodos fitoparásitos por centrifugación en azúcar. Boletín del Servicio de Plagas Forestales, v.9, p.183-189, 1983.

PARDO, A.; DE JUAN, J.A.; PARDO, J.E. Bacterial activity in different types of casing during mushroom cultivation Agaricus bisporus (Lange) Imbach. Acta Alimentaria, v.31, p.327-342, 2002.

PARDO, A.; NAVARRO, M.J.; LÓPEZ, M.J.; GEA, F.J. Uso del compost agotado de hongos cultivados reciclado como material de cobertura para el cultivo de champiñón. In: CONGRESO DE LA SOCIEDAD ESPAÑOLA DE AGRICULTURA ECOLÓGICA, 2., 2004, Almería. Agroecología: referente para la transición de los sistemas agrarios. Catarroja: Valencia, 2004. p.1599-1609.

PARDO, A.; PARDO, J.E. Evaluation of casing materials made from spent mushroom substrate and coconut fibre pith for use in 
production of Agaricus bisporus (Lange) Imbach. Spanish Journal of Agricultural Research, v.6, p.683-690, 2008.

PARDO,A.; PARDO, J.E.; DEJUAN, J.A. Cobertura y fructificación del champiñón cultivado, Agaricus bisporus (Lange) Imbach: materiales y aspectos prácticos. In: JORNADAS TÉCNICAS DEL CHAMPINOON Y OTROS HONGOS COMESTIBLES EN CASTILlA-LA MANCHA, 2., 1997, Cuenca. Anales. Cuenca: Diputación Provincial de Cuenca, 1999. p.101-130.

PÉREZ-CLAVIJO, M.; PEÑA-DÍAZ, M.L.; GRIJALBA-GARRIDO, S. Tierras de cobertura en el cultivo del champiñón: materiales alternativos y sostenibles. Actas de Horticultura, v.54, p.659-663, 2009.

RIAHI, H.; ARAB, A. Spent mushroom compost as an alternative for casing soil. Mushroom Science, v.16, p.585-589, 2004.

RIAHI, H.; ZAMANI, H. Use of spent mushroom compost and composted azolla as an alternative for casing soil. Mushroom Science, v.17, p.333-339, 2008.
RINKER, D.L. Handling and using "spent" mushroom substrate around the world. In: SÁNCHEZ, J.E.; HUERTA, G.; MONTIEL, E. (Ed.). Mushroom biology and mushroom products. Cuernavaca: Universidad Autónoma del Estado de Morelos, 2002. p.43-60.

SCHISLER, L.C. Quality/size. Mushroom Journal, v.123, p.81-89, 1983.

SINDEN, J.W. Ecological control of pathogens and weed-molds in mushroom culture. Annual Review of Phytopathology, v.9, p.411-432, 1971.

SINGH, M.; SINGH, R.P.; CHAUBE, H.S. Impact of physico-chemical properties of casing on yield of Agaricus bisporus (Lange) Imbach. Mushroom Science, v.15, p.441-446, 2000.

TELLO, J.; VARÉS, F.; LACASA, A. Selección y tratamiento de muestras: análisis de muestras, observación microscópica. In: MINISTERIO DEAGRICULTURA, PESCAY ALIMENTACIÓN. Manual de laboratorio: diagnóstico de hongos, bacterias y nematodos fitopatógenos. Madrid: MAPA, 1991. p.29-77.

Recibido el 12 de marzo de 2010 y aceptado el 29 de setembro de 2010 